Proyecciones Journal of Mathematics

Vol. 38, No 1, pp. 83-96, March 2019.

Universidad Católica del Norte

Antofagasta - Chile

\title{
3-difference cordiality of some corona graphs
}

\author{
R. Ponraj \\ Sri Paramakalyani College, India \\ M. Maria Adaickalam \\ District Statistical Office, India \\ and \\ R. Kala \\ Manonmaniam Sundaranar University, India \\ Received: August 201\%. Accepted : October 2018
}

\begin{abstract}
Let $G$ be a $(p, q)$ graph. Let $f: V(G) \rightarrow\{1,2, \ldots, k\}$ be a map where $k$ is an integer $2 \leq k \leq p$. For each edge uv, assign the label $|f(u)-f(v)| . \quad f$ is called $k$-difference cordial labeling of $G$ if $\left|v_{f}(i)-v_{f}(j)\right| \leq 1$ and $\left|e_{f}(0)-e_{f}(1)\right| \leq 1$ where $v_{f}(x)$ denotes the number of vertices labelled with $x, e_{f}(1)$ and $e_{f}(0)$ respectively denote the number of edges labelled with 1 and not labelled with 1 . A graph with a $k$-difference cordial labeling is called a $k$-difference cordial graph. In this paper we investigate 3-difference cordial labeling behavior of $T_{n} \odot K_{1}, T_{n} \odot 2 K_{1}, T_{n} \odot K_{2}, A\left(T_{n}\right) \odot K_{1}, A\left(T_{n}\right) \odot 2 K_{1}$, $A\left(T_{n}\right) \odot K_{2}$.
\end{abstract}

Subjclass 2000 : $05 C 78$.

Keywords : Cycle, path, triangular snake, quadrilateral snake, difference cordial. 


\section{Introduction}

Let $G$ be a $(p, q)$ simple, undirected, finite graph on $p$ vertices and $q$ edges. Graph labeling is used in several areas of science and technology like coding theory, astronomy, circuit design etc. For more details refer Gallian [1]. In 1987, Cahit [1] introduced the cordial labeling of graphs. Let $f$ be a function from the vertices of $G$ to $\{0,1\}$ and for each edge $x y$ assign the label $|f(x)-f(y)|$. Call $f$ a cordial labeling of $G$ if the number of vertices labeled 0 and the number of vertices labeled 1 differ by at most 1 , and the number of edges labeled 0 and the number of edges labeled 1 differ by at most 1 . Let $G_{1}, G_{2}$ respectively be $\left(p_{1}, q_{1}\right),\left(p_{2}, q_{2}\right)$ graphs. The corona of $G_{1}$ with $G_{2}, G_{1} \odot G_{2}$ is the graph obtained by taking one copy of $G_{1}$ and $p_{1}$ copies of $G_{2}$ and joining the $i^{t h}$ vertex of $G_{1}$ with an edge to every vertex in the $i^{t h}$ copy of $G_{2}$. The notion of difference cordial labeling was introduced by R. Ponraj, S. Sathish Narayanan and R. Kala in [4]. Seoud and Salman [10], studied the difference cordial labeling behavior of some families of graphs and they are ladder, triangular ladder, grid, step ladder and two sided step ladder graphs etc. Recently Ponraj et al. [4], introduced the concept of $k$-difference cordial labeling of graphs and studied the 3-difference cordial labeling behavior of star, $m$ copies of star etc. In $[5,6,7,8,9]$ they discussed the 3-difference cordial labeling behavior of path, cycle, complete graph, complete bipartite graph, star, bistar, comb, double comb, quadrilateral snake, wheel, helms, flower graph, sunflower graph, lotus inside a circle, closed helm, double wheel, union of graphs with the star, union of graphs with splitting graph of star, union of graphs with subdivided star, union of graphs with bistar, $P_{n} \cup P_{n},\left(C_{n} \odot K_{1}\right) \cup\left(C_{n} \odot K_{1}\right), F_{n} \cup F_{n}, K_{1, n} \odot K_{2}$,

$P_{n} \odot 3 K_{1}, m C_{4}, \operatorname{spl}\left(K_{1, n}\right), D S\left(B_{n, n}\right), C_{n} \odot K_{2}, C_{4}^{(t)}, S\left(K_{1, n}\right), S\left(B_{n, n}\right)$, $D A\left(T_{n}\right) \odot K_{1}, D A\left(T_{n}\right) \odot 2 K_{1}, D A\left(T_{n}\right) \odot K_{2}, D A\left(Q_{n}\right) \odot K_{1}, D A\left(Q_{n}\right) \odot 2 K_{1}$, triangular snake, alternate triangular snake, alternate quadrilateral snake, irregular triangular snake, irregular quadrilateral snake, double triangular snake, double quadrilaterla snake, double alternate triangular snake, and double alternate quadrilateral snake and some more graphs. In this paper we investigate 3 -difference cordial labeling behavior of $T_{n} \odot K_{1}, T_{n} \odot 2 K_{1}$, $T_{n} \odot K_{2}, A\left(T_{n}\right) \odot K_{1}, A\left(T_{n}\right) \odot 2 K_{1}, A\left(T_{n}\right) \odot K_{2}$. Terms are not defined here follows from Harary [2]. 


\section{2. $k$-Difference cordial labeling}

Definition 2.1. Let $G$ be a $(p, q)$ graph. Let $f: V(G) \rightarrow\{1,2, \ldots, k\}$ be a map. For each edge uv, assign the label $|f(u)-f(v)| . \quad f$ is called a $k$ difference cordial labeling of $G$ if $\left|v_{f}(i)-v_{f}(j)\right| \leq 1$ and $\left|e_{f}(0)-e_{f}(1)\right| \leq 1$ where $v_{f}(x)$ denotes the number of vertices labelled with $x, e_{f}(1)$ and $e_{f}(0)$ respectively denote the number of edges labelled with 1 and not labelled with 1 . A graph with a $k$-difference cordial labeling is called a $k$-difference cordial graph.

Example 2.1. An example of 3-difference cordial labeling is given in figure 1 .

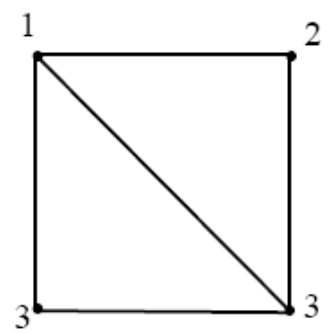

FIGURE 1

We investigate the 3-difference cordial labeling behavior of some path related graphs. The triangular snake $T_{n}$ is obtained from the path $P_{n}$ by replacing each edge of the path by a triangle $C_{3}$. Let $P_{n}$ be the path $u_{1} u_{2} \ldots u_{n}$. Let $V\left(T_{n}\right)=V\left(P_{n}\right) \cup\left\{v_{i}: 1 \leq i \leq n-1\right\}$ and $E\left(T_{n}\right)=$ $E\left(P_{n}\right) \cup\left\{u_{i} v_{i}, v_{i} u_{i+1}: 1 \leq i \leq n-1\right\}$. We now investigate the 3-difference cordiality of corona of triangular snakes $T_{n}$ with $K_{1}, 2 K_{1}$ and $K_{2}$.

Theorem 2.1. If $n \leq 13$ then $T_{n} \odot K_{1}$ is 3-difference cordial.

Proof. Clearly, $T_{n} \odot K_{1}$ has 4 n-2 vertices 5n-4 edges. Let $V\left(T_{n} \odot K_{1}\right)=$ $V\left(T_{n}\right) \cup\left\{w_{i}: 1 \leq i \leq n\right\} \cup\left\{z_{i}: 1 \leq i \leq n-1\right\}$ and $E\left(T_{n} \odot K_{1}\right)=$ $E\left(T_{n}\right) \cup\left\{u_{i} w_{i}: 1 \leq i \leq n\right\} \cup\left\{v_{i} z_{i}: 1 \leq i \leq n-1\right\}$. Consider the path vertices $u_{i}$. Assign the label 1 to the path vertices $u_{1}, u_{2}, u_{3}, \ldots$ Then assign the label 2 to all the vertices $v_{i}(1 \leq i \leq n-1)$. Assign the label 3 to all 
the vertices of $w_{i}(1 \leq i \leq n)$. Next we move to the vertices $w_{i}$. Assign the label 3 and 2 to the vertices $w_{6}$ and $w_{7}$ respectively. Then assign the label 1 to the vertices $z_{7 i+1}, z_{7 i+5}$ for $\mathrm{i}=0,1$. Assign the label 2 to the vertices $z_{7 i+2}, z_{7 i+4}$ for $\mathrm{i}=0,1$. For the values of $\mathrm{i}=0,1$ assign the label 3 to the vertices $z_{7 i+3}$. The vertex and edge conditions are given in table 2.1 and table 2.2 .

\begin{tabular}{|c|c|c|}
\hline Nature of $\mathrm{n}$ & $e_{f}(0)$ & $e_{f}(1)$ \\
\hline $\mathrm{n}=3,5$ & $\frac{5 n-3}{2}$ & $\frac{5 n-5}{2}$ \\
\hline $\mathrm{n}=4,6,8,10,12$ & $\frac{5 n-4}{2}$ & $\frac{5 n-4}{2}$ \\
\hline $\mathrm{n}=7,9,11,13$ & $\frac{5 n-5}{2}$ & $\frac{5 n-3}{2}$ \\
\hline
\end{tabular}

Table 2.1:

\begin{tabular}{|c|c|c|c|}
\hline values of $\mathrm{n}$ & $v_{f}(1)$ & $v_{f}(2)$ & $v_{f}(3)$ \\
\hline $\mathrm{n}=5,8,11$ & $\frac{4 n-2}{3}$ & $\frac{4 n-2}{3}$ & $\frac{4 n-2}{3}$ \\
\hline $\mathrm{n}=3,6,9$ & $\frac{4 n}{3}$ & $\frac{4 n-3}{3}$ & $\frac{4 n-3}{3}$ \\
\hline $\mathrm{n}=4,7$ & $\frac{4 n-1}{3}$ & $\frac{4 n-4}{3}$ & $\frac{4 n-1}{3}$ \\
\hline $\mathrm{n}=10,13$ & $\frac{4 n-1}{3}$ & $\frac{4 n-1}{3}$ & $\frac{4 n-4}{3}$ \\
\hline $\mathrm{n}=12$ & $\frac{4 n-3}{3}$ & $\frac{4 n}{3}$ & $\frac{4 n-3}{3}$ \\
\hline
\end{tabular}

Table 2.2:

Example 2.2. A 3-difference cordial labeling of $T_{6} \odot K_{1}$ is given in figure 2.

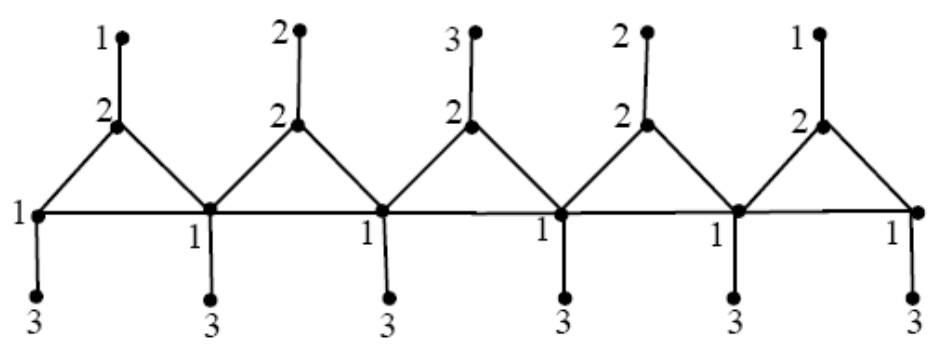

FIGURE 2 
Theorem 2.2. $T_{n} \odot 2 K_{1}$ is 3-difference cordial for all values of $n$.

Proof. Clearly the order an size of $T_{n} \odot 2 K_{1}$ are $6 \mathrm{n}-3$ and $7 \mathrm{n}-5$ respectively. Let $V\left(T_{n} \odot 2 K_{1}\right)=V\left(T_{n}\right) \cup\left\{w_{i}, w_{i}^{\prime}: 1 \leq i \leq n\right\} \cup\left\{z_{i}, z_{i}^{\prime}: 1 \leq i \leq\right.$ $n-1\}$ and $E\left(T_{n} \odot 2 K_{1}\right)=E\left(T_{n}\right) \cup\left\{u_{i} w_{i}, u_{i} w_{i}^{\prime}: 1 \leq i \leq n\right\} \cup\left\{v_{i} z_{i}, v_{i} z_{i}^{\prime}:\right.$ $1 \leq i \leq n-1\}$. Consider the path vertices $u_{i}$ as in the label 2 to the vertices $u_{1}, u_{3}, u_{5}, \ldots$ Assign the label 3 to the vertices $u_{2}, u_{4}, u_{6}, \ldots$ We now consider the vertices $v_{i}$. Assign the label 2 to the vertices $v_{1}$. Then assign the label 1 to the vertices $v_{2}, v_{3}, v_{4}, \ldots$ Next we move to the vertices $z_{i}$ and $z_{i}^{\prime}$. Assign the label 1 and 4 to all the vertices of $z_{i}(1 \leq i \leq n-1)$ and $z_{i}^{\prime}(1 \leq i \leq n-1)$ respectively. Consider the vertices $w_{i}$ and $w_{i}^{\prime}$. Assign the labels 1,1 to the vertices $w_{1}, w_{2}$ respectively and assign the labels 2,3 to the vertices $w_{1}^{\prime}, w_{2}^{\prime}$ respectively. Now we assign the label 2 to the vertices $w_{3}, w_{4}, w_{5}, \ldots$. Then assign the label 3 to the vertices $w_{3}^{\prime}, w_{5}^{\prime}, w_{7}^{\prime}, \ldots$ Finally assign the label 2 to the vertices $w_{4}^{\prime}, w_{6}^{\prime}, w_{8}^{\prime}, \ldots$. The vertex and edge condition is given by $v_{f}(1)=v_{f}(2)=v_{f}(3)=2 n-1$.

\begin{tabular}{|cc|c|c|}
\hline \multicolumn{2}{|c|}{ Nature of $\mathrm{n}$} & $e_{f}(0)$ & $e_{f}(1)$ \\
\hline$n \equiv 1 \quad(\bmod 2)$ & $\frac{7 n-5}{2}$ & $\frac{7 n-5}{2}$ \\
\hline$n \equiv 0 \quad(\bmod 2)$ & $\frac{7 n-6}{2}$ & $\frac{7 n-4}{2}$ \\
\hline
\end{tabular}

Table 2.3:

Theorem 2.3. $T_{n} \odot K_{2}$ is 3-difference cordial for all values of $n$.

Proof. Clearly the order an size of $T_{n} \odot K_{2}$ are $6 \mathrm{n}-3$ and $7 \mathrm{n}-5$ respectively. Let $V\left(T_{n} \odot K_{2}\right)=V\left(T_{n}\right) \cup\left\{w_{i}, w_{i}^{\prime}: 1 \leq i \leq n\right\} \cup\left\{z_{i}, z_{i}^{\prime}: 1 \leq i \leq n-1\right\}$ and $E\left(T_{n} \odot K_{2}\right)=E\left(T_{n}\right) \cup\left\{u_{i} w_{i}, u_{i} w_{i}^{\prime}, w_{i} w_{i}^{\prime}: 1 \leq i \leq n\right\} \cup\left\{v_{i} z_{i}, v_{i} z_{i}^{\prime}, z_{i} z_{i}^{\prime}\right.$ : $1 \leq i \leq n-1\}$.

Case 1. $n \equiv 1 \quad(\bmod 4)$. First we consider the path vertices $u_{i}$. Assign the label 1 to the all path vertices $u_{i}(1 \leq i \leq n)$. Then we move to the vertices $v_{i}$. Assign the label 1,1,1,2 to the first four vertices $v_{1}, v_{2}, v_{3}, v_{4}$ respectively. Then we assign the label $1,1,1,2$ to the next four vertices $v_{5}, v_{6}, v_{7}, v_{8}$ respectively. Continue this process we assign the next four vertices and so on. Note that in this case th last vertex received the label 2. Consider the vertices $w_{i}$ and $w_{i}^{\prime}$. Assign the lbel 2 to all the vertices $w_{i}$ $(1 \leq i \leq n)$ and assign the label 3 to all the vertices of $w_{i}^{\prime}(1 \leq i \leq n)$. Next 
we move to the vertices $z_{i}$ and $z_{i}^{\prime}$. Assign the label 2,2,2,1 to the first four vertices $z_{1}, z_{2}, z_{3}, z_{4}$ respectively. Then we assign the labels $2,2,2,1$ to the next four vertices $z_{5}, z_{6}, z_{7}, z_{8}$ respectively. Proceeding like this we assign the label to the next four vertices and so on. Clearly the last vertex $z_{n-1}$ received the label 1. Assign the label 3 to all the vertices of $z_{i}(1 \leq i \leq$ $n-1)$. The vertex and edge condition is given by $v_{f}(1)=v_{f}(2)=v_{f}(3)=$ $2 n-1$ and $e_{f}(0)=\frac{9 n-7}{2}$ and $e_{f}(1)=\frac{9 n-5}{2}$.

Case 2. $n \equiv 2 \quad(\bmod 4)$. Assign the label to the vertices $u_{i}, w_{i}$ and $w_{i}^{\prime}$ $(1 \leq i \leq n)$ as in case 1 . Consider the vertices $v_{i}$. Assign the label 1 to the vertex $v_{1}$. Now we assign the labels $1,1,1,2$ to the vertices $v_{2}, v_{3}, v_{4}, v_{5}$ respectively. Then we assign the labels $1,1,1,2$ to the next four vertices $v_{6}, v_{7}, v_{8}, v_{9}$ respectively. Continuing like this assign the label to the next four vertices and so on. Note that in this case the last vertex $v_{n-1}$ received the label 2. Now we move to the vertices $z_{i}$ and $z_{i}^{\prime}$. Assign the label to the vertices $z_{i}^{\prime}(1 \leq i \leq n-1)$ as in case 1 . Then we assign the label 2 to the vertex $z_{1}$. Now we assign the labels $2,2,2,1$ to the four vertices $z_{2}, z_{3}, z_{4}, z_{5}$ respectively. Then assign the labels $2,2,2,1$ to the next four vertices $z_{6}, z_{7}, z_{8}, z_{9}$ respectively. Proceeding like this assign the label to the next four vertices and so on. Clearly the last vertex $z_{n-1}^{\prime}$ received the label 1. The vertex and edge condition of this case is $v_{f}(1)=v_{f}(2)=$ $v_{f}(3)=2 n-1$ and $e_{f}(0)=e_{f}(1)=\frac{9 n-6}{2}$.

Case 3. $n \equiv 3 \quad(\bmod 4)$. Consider the path vertices $u_{i}$. Assign the label to the vertices $u_{i}(1 \leq i \leq n)$ as in case 1 . Next we assign the labels 1,1 to the vertices $v_{1}, v_{2}$ respectively. Assign the labels 1,1,1,2 to the four vertices $v_{3}, v_{4}, v_{5}, v_{6}$ respectively. Then we assign the labels $1,1,1,2$ to the next four vertices $v_{7}, v_{8}, v_{9}, v_{10}$ respectively. Continuing like this assign the label to the next four vertices and so on. Note that in this case the last vertex $v_{n-1}$ received the label 2. Assign the label to the vertices $w_{i}(1 \leq i \leq n)$ and $w_{i}^{\prime}$ $(1 \leq i \leq n)$ as in case 1 . Now we move to the vertices $z_{i}$ and $z_{i}^{\prime}$. Assign the labels 2,2 to the vertices $z_{1}, z_{2}$ respectively. Next we assign the label $2,2,2,1$ to the vertices $z_{3}, z_{4}, z_{5}, z_{6}$ respectively. Then we assign the label 2,2,2,1 to the vertices $z_{7}, z_{8}, z_{9}, z_{10}$ respectively. Proceeding like this assign the label to the next four vertices and so on. Clearly the last vertex $z_{n-1}$ received the label 1. Assign the label $z_{i}^{\prime}$ as in case 1 . The vertex and edge condition is $v_{f}(1)=v_{f}(2)=v_{f}(3)=2 n-1$ and $e_{f}(0)=\frac{9 n-5}{2}$ and $e_{f}(1)=\frac{9 n-7}{2}$. 
Example 2.3. A 3-difference cordial labeling of $T_{5} \odot K_{2}$ is given in figure 3.

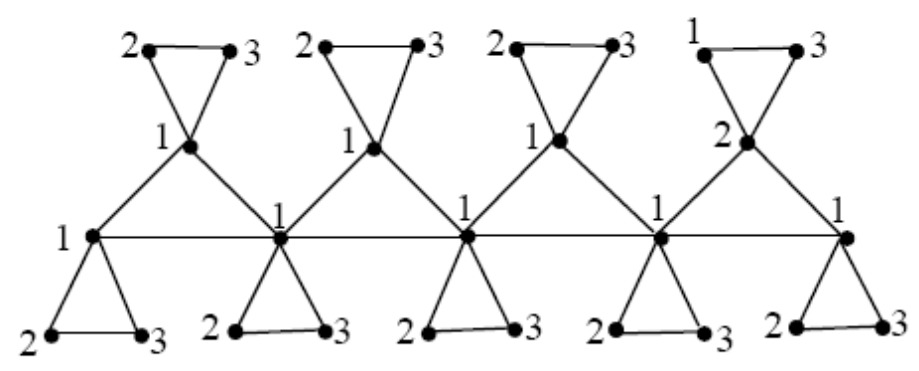

FIGURE 3

An alternate triangular snake $A\left(T_{n}\right)$ is obtained from a path $u_{1} u_{2} \ldots u_{n}$ by joining $u_{i}$ and $u_{i+1}$ (alternatively) to new vertex $v_{i}$. That is every alternate edge of a path is replaced by $C_{3}$.

Theorem 2.4. $A\left(T_{n}\right) \odot K_{1}$ is 3 -difference cordial for all values of $n$.

Proof. Case 1. Let the first triangle starts from $u_{1}$ and the last triangle ends with $u_{n}$. Here $n$ is even. Let $V\left(A\left(T_{n}\right) \odot K_{1}\right)=V\left(A\left(T_{n}\right)\right) \cup\left\{x_{i}: 1 \leq\right.$ $i \leq n\} \cup\left\{w_{i}: 1 \leq i \leq \frac{n}{2}\right\}$ and $E\left(A\left(T_{n}\right) \odot K_{1}\right)=E\left(A\left(T_{n}\right)\right) \cup\left\{u_{i} x_{i}: 1 \leq i \leq\right.$ $n\} \cup\left\{v_{i} w_{i}: 1 \leq i \leq \frac{n}{2}\right\}$. In this case the order and size of $A\left(T_{n}\right) \odot K_{1}$ are $3 \mathrm{n}$ and $\frac{7 n-2}{2}$. First we consider the path vertices $u_{i}$. Assign the labels $1,1,2,1$ to the vertices $u_{1}, u_{2}, u_{3}, u_{4}$ respectively. Next we assign the labels $2,1,1,1$ to the vertices $u_{5}, u_{6}, u_{7}, u_{8}$ respectively. Then we assign the labels $2,1,1,1$ to the vertices $u_{9}, u_{10}, u_{11}, u_{12}$ respectively. Continuing this way assign the label to the next four vertices and so on. Clearly the last vertex $u_{n}$ received the label 1. Consider the vertices $v_{i}$. Assign the label 1 to the vertices $v_{1}, v_{3}, v_{5}, v_{7} \ldots$ Then we assign the label 2 to the vertices $v_{2}, v_{4}, v_{6}, v_{8} \ldots$ Now we assign the label 3 to the vertices $w_{i}\left(1 \leq i \leq \frac{n}{2}\right)$. Next we move to the vertices $x_{i}$. Assign the labels 2,2,3,3 to the vertices $x_{1}, x_{2}, x_{3}, x_{4}$ respectively. Then we assign the label 2 to the vertices $x_{5}, x_{7}, x_{9}, x_{11} \ldots$ Finally assign the label 3 to the vertices $x_{6}, x_{8}, x_{10}, x_{12} \ldots$ The vertex and edge condition of this case is $v_{f}(1)=v_{f}(2)=v_{f}(3)=n$ and 


\begin{tabular}{|cc|c|c|}
\hline \multicolumn{2}{|c|}{ Nature of $\mathrm{n}$} & $e_{f}(0)$ & $e_{f}(1)$ \\
\hline$n \equiv 2 \quad(\bmod 4)$ & $\frac{7 n-2}{4}$ & $\frac{7 n-2}{4}$ \\
\hline$n \equiv 0 \quad(\bmod 4)$ & $\frac{7 n-4}{4}$ & $\frac{7 n}{4}$ \\
\hline
\end{tabular}

Table 2.4:

Case 2. Let the first triangle starts from $u_{2}$ and the last triangle ends with $u_{n-1}$. Here also $n$ is even. In this case the order and size of $A\left(T_{n}\right) \odot$ $K_{1}$ are $3 n-2$ and $\frac{7 n-8}{2}$. First we consider the path vertices $u_{i}$. Assign the labels $1,1,1$ to the vertices $u_{1}, u_{2}, u_{3}$ respectively. Now we assign the labels $1,1,1,2$ to the vertices $u_{4}, u_{5}, u_{6}, u_{7}$ respectively. Then we assign the labels $1,1,1,2$ to the vertices $u_{8}, u_{9}, u_{10}, u_{11}$ respectively. Proceeding like this assign the label to the next four vertices and so on. Note that in this case the last vertex $u_{n}$ received the label 1 . Next we move to the vertices $v_{i}$. Assign the label 2 to the vertex $v_{1}$. Then assign the label 2 to the vertices $v_{2}, v_{4}, v_{6}, v_{8}, \ldots$ and assign the label 1 to the vertices $v_{3}, v_{5}, v_{7}, v_{9}, \ldots$ Consider the vertices $w_{i}$. Assign the label to the vertices $w_{i}\left(1 \leq i \leq \frac{n}{2}\right)$ as in case 1 . Now we move to the vertices $x_{i}$. Assign the label 2 to the vertices $x_{1}, x_{2}, x_{5}, x_{7}$ and assign the label 3 to the vertices $x_{3}, x_{4}, x_{6}$. Assign the label 3 to all the vertices $x_{4 i}$ and $x_{4 i+1}$ for all the values of $\mathrm{i}=2,3,4, \ldots$ For all the values of $\mathrm{i}=2,3,4, \ldots$ assign the label to the vertices $x_{4 i+2}$ and $x_{4 i+3}$. Clearly the last four vertices $x_{n-3}, x_{n-2}, x_{n-1}, x_{n}$ received the label by the integers $2,2,3,3$. The vertex condition is $v_{f}(2)=v_{f}(3)=n-1$ and $v_{f}(1)=n$. Also the edge condition is $v_{f}(2)=v_{f}(3)=n-1$ and $v_{f}(1)=n$. Also the edge condition is given in table 2.5.

\begin{tabular}{|cc|c|c|}
\hline \multicolumn{2}{|c|}{ Nature of $\mathrm{n}$} & $e_{f}(0)$ & $e_{f}(1)$ \\
\hline$n \equiv 0 \quad(\bmod 4)$ & $\frac{7 n-8}{4}$ & $\frac{7 n-8}{4}$ \\
\hline$n \equiv 2 \quad(\bmod 4)$ & $\frac{7 n-10}{4}$ & $\frac{7 n-6}{4}$ \\
\hline
\end{tabular}

Table 2.5:

Case 3. Let the first triangle starts from $u_{2}$ and the last triangle ends with $u_{n}$. Here $n$ is odd. In this case the order and size of $A\left(T_{n}\right) \odot K_{1}$ are $3 \mathrm{n}-1$ and $\frac{7 n-5}{2}$ respectively. We consider the path vertices $u_{i}$. Assign the label 1 to the vertices $u_{1}, u_{2}$. Now we assign the labels $1,1,1,2$ to the vertices $u_{3}, u_{4}, u_{5}, u_{6}$ respectively. Then we assign the labels $1,1,1,2$ to the vertices $u_{7}, u_{8}, u_{9}, u_{10}$ respectively. Continuing this way assign the label to the next four vertices and so on. Note that in this case the last vertex $u_{n}$ received the label 1 . Next we move to the vertices $v_{i}$. Assign the label to 
the vertices $v_{i}\left(1 \leq i \leq\left\lceil\frac{n}{2}\right\rceil\right)$ as in case 2 . We consider the vertices $w_{i}$. Assign the lbel to the vertices $w_{i}$ as in case 1 . Next we move to the vertices $x_{i}$. Assign the label 3 to th vertices $x_{1}$ and $x_{2}$. Then we assign the label 2 to the vertex $x_{3}$. Assign the label 3 to the vertices $x_{4 i}, x_{4 i+3}$ for all the values of $i=1,2,3 \ldots$ For all the values of $i=1,2,3, \ldots$ assign the label 2 to the vertices $x_{4 i+1}$ and $x_{4 i+2}$. Note that in this process the last four vertices $x_{n-3}, x_{n-2}, x_{n-1}, x_{n}$ received the label by the integers $3,2,2,3$. The vertex condition is $v_{f}(2)=n-1$ and $v_{f}(1)=v_{f}(3)=n$. Also the edge condition is given in table 2.6 .

\begin{tabular}{|cc|c|c|}
\hline \multicolumn{2}{|c|}{ Values of $\mathrm{n}$} & $e_{f}(0)$ & $e_{f}(1)$ \\
\hline$n \equiv 3 \quad(\bmod 4)$ & $\frac{7 n-5}{4}$ & $\frac{7 n-5}{4}$ \\
\hline$n \equiv 1 \quad(\bmod 4)$ & $\frac{7 n-7}{4}$ & $\frac{7 n-3}{4}$ \\
\hline
\end{tabular}

Table 2.6:

Theorem 2.5. $A\left(T_{n}\right) \odot 2 K_{1}$ is 3-difference cordial.

Proof. Case 1. Let the first triangle starts from $u_{1}$ and the last triangle ends with $u_{n}$. Here $n$ is even. Let $V\left(A\left(T_{n}\right) \odot 2 K_{1}\right)=V\left(A\left(T_{n}\right)\right) \cup\left\{x_{i}, x_{i}^{\prime}\right.$ : $1 \leq i \leq n\} \cup\left\{w_{i}, w_{i}^{\prime}: 1 \leq i \leq \frac{n}{2}\right\}$ and $E\left(A\left(T_{n}\right) \odot 2 K_{1}\right)=E\left(A\left(T_{n}\right)\right) \cup$ $\left\{u_{i} x_{i}, u_{i} x_{i}^{\prime}: 1 \leq i \leq n\right\} \cup\left\{v_{i} w_{i}, v_{i} w_{i}^{\prime}: 1 \leq i \leq \frac{n}{2}\right\}$. In this case the order and size of $A\left(T_{n}\right) \odot 2 K_{1}$ are $\frac{9 n}{2}$ and $5 n-1$ respectively. Assign the labels 1,1 to the vertices $u_{1}, u_{2}$ respectively. Then we assign the path vertices $u_{3}, u_{5}, u_{7}, u_{9} \ldots$ labeled by the integer 1 . Now we assign the label 2 to the vertices $u_{4}, u_{6}, u_{8}, u_{10} \ldots$ Consider the vertices $v_{i}$. Assign the label 2 to the vertex $v_{1}$. Then assign the label 1 to the vertices $v_{2}, v_{3}, v_{4}, v_{5} \ldots$ Now we move to the vertices $w_{i}$ and $w_{i}^{\prime}$. Assign the 1 to all the vertices of $w_{i}$ $\left(1 \leq i \leq \frac{n}{2}\right)$ and assign the label 3 to all the vertices of $w_{i}^{\prime}\left(1 \leq i \leq \frac{n}{2}\right)$. Finally we consider the vertices $w_{i}$ and $w_{i}^{\prime}$. Assign the label 2 to all the vertices of $x_{i}(1 \leq i \leq n)$. Next we assign the label 3 to all the vertices of $x_{i}^{\prime}\left(1 \leq i \leq \frac{n}{2}\right)$. The vertex condition is $v_{f}(1)=v_{f}(2)=v_{f}(3)=\frac{3 n}{2}$ and $e_{f}(0)=\frac{5 n-2}{2}$ and $e_{f}(1)=\frac{5 n}{2}$. 
Case 2. Let the first triangle starts from $u_{2}$ and the last triangle ends with $u_{n-1}$. Here $n$ is even. In this case the order and size of $A\left(T_{n}\right) \odot 2 K_{1}$ are $\frac{9 n-6}{2}$ and $5 n-5$ respectively. First we consider the path vertices $u_{i}$. Assign the label 1 to the vertices $u_{1}, u_{2}, u_{3}$ respectively. Then we assign the label 2 to the vertices $u_{5}, u_{7}, u_{9}, u_{11}, \ldots$ Next we assign the label 1 to the vertices $u_{4}, u_{6}, u_{8}, u_{10} \ldots$ Note that in this case the last vertex $u_{n}$ received the label 1. Then we move to the vertices $v_{i}, w_{i}, w_{i}^{\prime}, x_{i}$ and $x_{i}^{\prime}$. Assign the label to the vertices $v_{i}(1 \leq i \leq n-1), w_{i}\left(1 \leq i \leq \frac{n}{2}\right), w_{i}^{\prime}\left(1 \leq i \leq \frac{n}{2}\right), x_{i}$ $(1 \leq i \leq n)$ and $x_{i}^{\prime}(1 \leq i \leq n)$ as in case 1 . The vertex and edge condition of this case is given by $v_{f}(1)=v_{f}(2)=v_{f}(3)=\frac{9 n-6}{6}$ and $e_{f}(0)=\frac{5 n-6}{2}$ and $e_{f}(1)=\frac{5 n-4}{2}$.

Case 3. Let the first triangle starts from $u_{2}$ and the last triangle ends with $u_{n}$. Here $n$ is odd. In this case the order and size of $A\left(T_{n}\right) \odot 2 K_{1}$ are $\frac{9 n-3}{2}$ and $5 \mathrm{n}-3$ respectively. Consider the path vertices $u_{i}$. Assign the label 1 to the vertices $u_{1}, u_{3}, u_{5}, \ldots$. Then we assign the label 2 to the vertices $u_{2}, u_{4}, u_{6}, u_{8}, \ldots$ Clearly the last vertex $u_{n}$ received the label 1 . Next we consider the vertices $v_{i}$. Assign the label 1 to all the vertices of $v_{i}(1 \leq i \leq n-1)$. Next we move to the vertices $w_{i}, w_{i}^{\prime}, x_{i}$ and $x_{i}^{\prime}$. Assign the label to the vertices $w_{i}, w_{i}^{\prime}\left(1 \leq i \leq \frac{n}{2}\right)$ and $x_{i}, x_{i}^{\prime}(1 \leq i \leq n)$ as in case 1. Since $v_{f}(1)=v_{f}(2)=v_{f}(3)=\frac{9 n-3}{6}$ and $e_{f}(0)=e_{f}(1)=\frac{5 n-3}{2}$, this labeling is a 3 -difference cordial labeling.

Theorem 2.6. $A\left(T_{n}\right) \odot K_{2}$ is 3-difference cordial.

Proof. Case 1. Let the first triangle starts from $u_{1}$ and the last triangle ends with $u_{n}$. Here $n$ is even. Let $V\left(A\left(T_{n}\right) \odot K_{2}\right)=V\left(A\left(T_{n}\right)\right) \cup\left\{x_{i}, x_{i}^{\prime}\right.$ : $1 \leq i \leq n\} \cup\left\{w_{i}, w_{i}^{\prime}: 1 \leq i \leq \frac{n}{2}\right\}$ and $E\left(A\left(T_{n}\right) \odot K_{2}\right)=E\left(A\left(T_{n}\right)\right) \cup$ $\left\{u_{i} x_{i}, u_{i} x_{i}^{\prime}, x_{i} x_{i}^{\prime}: 1 \leq i \leq n\right\} \cup\left\{v_{i} w_{i}, v_{i} w_{i}^{\prime}, w_{i} w_{i}^{\prime}: 1 \leq i \leq \frac{n}{2}\right\}$. In this case the order and size of $A\left(T_{n}\right) \odot K_{2}$ are $\frac{9 n}{2}$ and $\frac{13 n-2}{2}$ respectively. Consider the path vertices $u_{i}$. Assign the label 1 to the vertices $u_{1}, u_{2}$ respectively. Assign the labels $1,1,2,1$ to the path vertices $u_{3}, u_{4}, u_{5}, u_{6}$ respectiely. Then we assign the labels $1,1,2,1$ to the next four path vertices $u_{7}, u_{8}, u_{9}, u_{10}$ respectively. Continuing like this we assign the label to the next four vertices and so on. Note that if all the vertices of $u_{i}$ are labeled then we stop. Otherwise there are some non labeled vertices are exists. If th number of non labeled vertices are less than or equal to 3 then assign the labels 1,1,2 to the non labeled vertices. If it is two then we assign the labels 1,1 to the non lbeled vertices. If only one non labeled vertex is exist then assign the label 1 only. Next we assign the label 2 to all the vertices of $v_{i}(1 \leq i \leq n-1)$. 
Consider the vertices $w_{i}$ and $w_{i}^{\prime}$. Assign the labels 2,3 to the vertices $w_{1}, w_{1}^{\prime}$ respectivly. Assign the label 2 to the vertices $w_{2}, w_{4}, w_{6} \ldots$ and assign the label 1 to the vertices $w_{3}, w_{5}, w_{7} \ldots$ Then we asign the label 3 to all the vertices of $w_{i}^{\prime}\left(1 \leq i \leq \frac{n}{2}\right)$. Next we move to the vertices $x_{i}$ and $x_{i}^{\prime}$. Assign the label 2 to the vertices $x_{3}, x_{3}, x_{5} \ldots$ Then we assign the label 1 to the vertices $x_{2}, x_{4}, x_{6} \ldots$ Finally we asign the label 3 to all the vertices of $x_{i}^{\prime}(1 \leq i \leq n)$. The vertex condition is $v_{f}(1)=v_{f}(2)=v_{f}(3)=\frac{3 n}{2}$. The edge condition is given in table 2.7 .

\begin{tabular}{|c|c|c|}
\hline Values of $n$ & $\bar{e}(0)$ & $e_{f}(1)$ \\
\hline$n \equiv 0 \quad(\bmod 4)$ & T: & $13 n-$ \\
\hline$n \equiv 2 \quad(\bmod 4)$ & $\underline{13 n-2}$ & $\frac{13 n-}{4}$ \\
\hline
\end{tabular}

Table 2.7:

Case 2. Let the first triangle starts from $u_{2}$ and the last triangle ends with $u_{n-1}$. Here $n$ is even. In this case the order and size of $A\left(T_{n}\right) \odot K_{2}$ are $\frac{9 n-6}{2}$ and $\frac{13 n-12}{2}$ respectively. First we consider the path vertices $u_{i}$. Assign the label 1 to the vertex $u_{1}$. Now we assign the labels 1,1,1,2 to the path vertices $u_{2}, u_{3}, u_{4}, u_{5}$ respectiely. Then we assign the labels $1,1,1,2$ to the next four path vertices $u_{6}, u_{7}, u_{8}, u_{9}$ respectively. Proceeding like this we assign the label to the next four vertices and so on. If all the vertices of $u_{i}$ are labeled then we stop the process. Otherwise there are some non labeled vertices are exists. If th number of non labeled vertices are less than or equal to 3 then assign the labels 1,1,1 to the non labeled vertices. If it is two then we assign the labels 1,1 to the non labeled vertices. If only one non labeled vertex is exist then assign the label 1 only. Next we assign the label 2 to all the vertices of $v_{i}$. Assign the label to the vertices $v_{i}(1 \leq i \leq n-1)$ as in case 1 . Now we move to the vertices $w_{i}$ and $w_{i}^{\prime}$. Assign the label 2 to the vertices $w_{1}, w_{3}, w_{5} \ldots$ and assign the label 1 to the vertices $w_{2}, w_{4}, w_{6} \ldots$ Then we assign the labels to the vertices $w_{i}^{\prime}$ $\left(1 \leq i \leq \frac{n}{2}\right)$ as in case 1 . Then we move to the vertices $x_{i}$ and $x_{i}^{\prime}$. Assign the label 2 to the vertices $x_{1}$ and $x_{2}$ respectively. Then we assign the label to the vertices $x_{i}^{\prime}(1 \leq i \leq n)$ as in case 1 . Next we assign the label 2 to the vertices $x_{2 i+1}$ for all the values of $i=1,2,3, \ldots$ For all the values of $i=1,2,3, \ldots$ assign the label 1 to the vertices $x_{2 i+2}$. The vertex condition of this case is $v_{f}(1)=v_{f}(2)=v_{f}(3)=\frac{3 n-2}{2}$. Also the edge condition of this case is given in table 2.8 . 


\begin{tabular}{|cc|c|c|}
\hline \multicolumn{2}{|c|}{ Nature of $\mathrm{n}$} & $e_{f}(0)$ & $e_{f}(1)$ \\
\hline$n \equiv 0 \quad(\bmod 4)$ & $\frac{13 n-12}{4}$ & $\frac{13 n-12}{4}$ \\
\hline$n \equiv 2$ & $(\bmod 4)$ & $\frac{13 n-14}{4}$ & $\frac{13 n-10}{4}$ \\
\hline
\end{tabular}

Table 2.8:

Case 3. Let the first triangle starts from $u_{2}$ and the last triangle ends with $u_{n}$. Here $n$ is oddd. In this case the order and size of $A\left(T_{n}\right) \odot K_{2}$ are $\frac{9 n-3}{2}$ and $\frac{13 n-7}{2}$ respectively. First we consider the path vertices $u_{i}$. Assign the label 1 to the vertex $u_{1}, u_{2}, u_{3}$. Next we assign the labels $1,1,2,1$ to the path vertices $u_{4}, u_{5}, u_{6}, u_{7}$ respectively. Then we assign the labels $1,1,2,1$ to the next four path vertices $u_{8}, u_{9}, u_{10}, u_{11}$ respectively. Continuing like this we assign the label to the next four vertices and so on. If all the vertices of $u_{i}$ are labeled then we stop the process. Otherwise there are some non labeled vertices are exists. If th number of non labeled vertices are less than or equal to 3 then assign the labels 1,1,2 to the non labeled vertices. If it is two then we assign the labels 1,1 to the non labeled vertices. If only one non labeled vertex is exist then assign the label 1 only. Consider the vertices of $v_{i}$. Assign the label 2 to the vertex $v_{1}$. Then we assign the label 2 to the vertices $v_{2}, v_{4}, v_{6} \ldots$ and assign the label 1 to the vertices $v_{3}, v_{5}, v_{7} \ldots$ Now we move to the vertices $w_{i}$ and $w_{i}^{\prime}$. Assign the label 2 to all the vertices of $w_{i}\left(1 \leq i \leq \frac{n}{2}\right)$ and assign the label 3 to all the vertices of $w_{i}^{\prime}\left(1 \leq i \leq \frac{n}{2}\right)$. Finally we consider the vertices $x_{i}$ and $x_{i}^{\prime}$. Assign the label 2 to the vertices $x_{1}$ and $x_{2}$. Next we assign the label to the vertices $x_{i}^{\prime}(1 \leq i \leq n)$ as in case 1 . Now we assign the label 1 to the vertices $x_{2 i+1}$ for all the values of $\mathrm{i}=1,2,3, \ldots$ and assign the label 2 to the vertices $x_{2 i+2}$ for all the values of $\mathrm{i}=1,2,3, \ldots$ The vertex condition is $v_{f}(1)=v_{f}(2)=v_{f}(3)=\frac{9 n-3}{6}$. Also the edge condition of this case is given in table 2.9.

\begin{tabular}{|cc|c|c|}
\hline \multicolumn{2}{|c|}{ Values of $\mathrm{n}$} & $e_{f}(0)$ & $e_{f}(1)$ \\
\hline$n \equiv 3 \quad(\bmod 4)$ & $\frac{13 n-7}{4}$ & $\frac{13 n-7}{4}$ \\
\hline$n \equiv 1 \quad(\bmod 4)$ & $\frac{13 n-5}{4}$ & $\frac{13 n-9}{4}$ \\
\hline
\end{tabular}

Table 2.9:

Example 2.4. A 3-difference cordial labeling of $A\left(T_{4}\right) \odot K_{2}$ with the first triangle starts from $u_{1}$ and the last triangle ends with $u_{n}$ is given in figure 4]. 


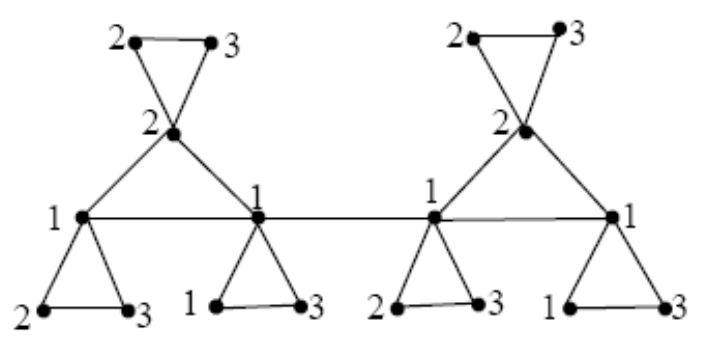

Figure 4

\section{References}

[1] Cahit.I, Cordial graph: a weaker version of graceful and harmonious graph, Ars Combinatoria, 23, pp. 201-207, (1987).

[2] J.A.Gallian, A Dynamic survey of graph labeling, The Electronic Journal of Combinatorics, 19 (2016). \#Ds6.

[3] F. Harary, Graph theory, Addision wesley, New Delhi (1969).

[4] R. Ponraj, S. Sathish Narayanan and R.Kala, Difference cordial labeling of graphs, Global Journal of Mathematical Sciences: Theory and Practical, 5, pp. 185-196, (2013).

[5] R. Ponraj, M. Maria Adaickalam and R.Kala, $k$-difference cordial labeling of graphs, International journal of mathematical combinatorics, 2, pp. 121-131, (2016).

[6] R. Ponraj, M. Maria Adaickalam, 3-difference cordial labeling of some union of graphs, Palestine journal of mathematics, 6 (1), pp. 202-210, (2017).

[7] R. Ponraj, M. Maria Adaickalam, 3-difference cordial labeling of cycle related graphs, Journal of algorithms and computation, 47, pp. 1-10, (2016). 
[8] R. Ponraj, M. Maria Adaickalam, 3-difference cordiality of some graphs, Palestine journal of mathematics, 2, pp. 141-148, (2017).

[9] R. Ponraj, M. Maria Adaickalam, 3-difference cordial labeling of corona related graphs, (communicated).

[10] R. Ponraj, M. Maria Adaickalam, and R.Kala, 3-difference cordial labeling of some path related graphs, (communicated).

[11] M. A.Seoud and Shakir M. Salman, On difference cordial graphs, Mathematica Aeterna, 5, No. 1, pp. 105-124, (2015).

\section{R. Ponraj}

Department of Mathematics

Sri Paramakalyani College,

Alwarkurichi-627412,

India

e-mail : ponrajmaths@gmail.com

\section{Maria Adaickalam}

Department of Economics and Statistics

District Statistical office,

Ramanathapuram-623501,

India

e-mail : mariaadaickalam@gmail.com

and

\section{R. Kala}

Department of Mathematics

Manonmaniam Sundaranar University,

Tirunelveli-627012,

India

e-mail : karthipyi91@yahoo.co.in 